\title{
Identification of natural splice variants of SAMHD1 in virus-infected HCC
}

\author{
YUNPENG SHI ${ }^{1}$, GUOYUE LV ${ }^{1}$, ZHE CHU $^{5}$, LILING PIAO ${ }^{3}$, XINGKAI LIU $^{1}$, \\ TUO WANG ${ }^{4}$, YANFANG JIANG ${ }^{2}$ and PING ZHANG ${ }^{1}$
}

\author{
${ }^{1}$ Department of Hepatobiliary and Pancreatic Surgery, The First Hospital of Jilin University; ${ }^{2}$ Key Laboratory of \\ Zoonosis Research, Ministry of Education, The First Hospital of Jilin University; ${ }^{3}$ Department of Endocrinology, \\ The Fourth Hospital of Jilin University; ${ }^{4}$ Hospital Management Section of Jilin University; \\ ${ }^{5}$ Department of Endocrinology, The First Hospital of Jilin University, Changchun, Jilin 130021, P.R. China
}

Received September 24, 2013; Accepted October 18, 2013

DOI: 10.3892/or.2013.2895

\begin{abstract}
It has been previously shown that the sterile alpha motif domain and HD domain-containing protein 1 (SAMHD1) can act as a retroviral restriction factor by inhibiting HIV-1 infection, but whether it has any roles in cancer is still unclear. In the present study, we identified several SAMHD1 splice variants naturally occurring in liver cancer and investigated their roles in regulating drug susceptibility. SAMHD1 variants were identified by sequencing. RT-PCR and western blot analysis were performed to verify the expression level of the polymorphisms. Cell cycle analysis was carried out using flow cytometry, and data were analyzed using Multicycle software. Several deletions of SAMHD1 were identified in both the patients and the healthy controls with no significant difference in respective frequencies, while an insertion in the exon 4 occurred at a higher frequency in HBV- and HCV-infected patients (36.4 and 30\%, respectively) when compared to the control groups. Following cisplatin treatment and cell cycle analysis, SAMHD1 variants showed different activities in increasing the susceptibility to chemotherapy drugs. The insertion of exon 4 correlated with the occurrence of virus infection in the HCC patients. In conclusion, analysis of the different activities of SAMHD1 splice variants in regulating drug sensitivity implied that the exon4 insertion might act as an indicator of the occurrence of liver cancer.
\end{abstract}

Correspondence to: Dr Ping Zhang, Department of Hepatobiliary and Pancreatic Surgery, The First Hospital of Jilin University, 71 Xinmin Street, Changchun, Jilin 130021, P.R. China

E-mail: pingzhang730@163.com

Dr Yanfang Jiang, Key Laboratory of Zoonosis Research, Ministry of Education, The First Hospital of Jilin University, Changchun, Jilin 130021, P.R. China

E-mail: yanfangjiang@hotmail.com

Key words: sterile alpha motif domain and HD domain-containing protein 1 , splice variants, hepatocellular carcinoma

\section{Introduction}

The sterile alpha motif domain and HD domain-containing protein 1 (SAMHD1) spans 59,523 bp of genomic sequence in 16 exons and encodes a 626 -amino acid protein. It is related to type 1 interferon and mediates a TNF- $\alpha$-related proinflammatory response in a series of autoimmune diseases, such as Aicardi-Goutières syndrome (AGS) (1-3) and systemic lupus erythematosus (SLE) (4). Recent reports have shown that SAMHD1 might function as a Vpx-sensitive retroviral restriction factor that inhibits HIV-1 infection in macrophages and dendritic cells through its intrinsic dGTP-dependent deoxynucleoside triphosphate (dNTP) hydrolase activity (5-7). Therefore, SAMHD1 might mediate its restriction activity by reducing the pool of dNTPs in virus-infected cells. This characteristic feature of SAMHD1 indicates that it might operate similarly in other diseases related to viral infections, such as nasopharyngeal carcinoma caused by Epstein-Barr virus (EB), cervical cancer caused by human papilloma virus (HPV) and liver cancer caused by hepatitis $\mathrm{B} / \mathrm{C}$ virus (HBV/HCV).

$\mathrm{HBV}$ infection is a major etiologic factor in the pathogenesis of hepatocellular carcinoma (HCC) $(8,9)$. Approximately 170 million individuals are chronically infected by HCV, and some of them develop progressive liver disease leading to cirrhosis and HCC (10-12). Despite identification of the main factors resulting in virus-mediated HCC, the roles of SAMHD1 in this disease are still unclear, and therefore we undertook a study of SAMHD1 in 44 patients with HCC and 10 healthy controls (HC). Although it has been reported that a number of SNPs in SAMHD1 are related to AGS syndrome or SLE, these mutations were found to occur at low frequencies in both groups (data not shown). In contrast, several splice variants of SAMHD1 exhibited high frequencies, including a novel insertion of exon4, deletions of exon8-9, 13, and $14(13,14)$. In the present study, we investigated the frequency of SAMHD1 splice variants, and compared their different activities in reversing sensitivity to chemotherapeutic drugs. Our results suggest that the exon4 insertion might act as an indicator of hepatocarcinogenesis. 


\section{Materials and methods}

Patients. Forty-four patients diagnosed with HCC by both surgical procedures and pathology participated in the present study in The First Hospital of Jilin University from 2009 to 2011. Since SAMHD1 is closely associated with the immune system, patients were selected with no previous history of autoimmune diseases to exclude from our results confounding contributions from other factors. The patients were divided into three groups: the HBV-infected group, the HCV-infected group, and the non-virus-infected group. Each patient in the virus-induced groups had been infected with the corresponding type of virus for more than seven years.

The 10 healthy controls (HC) were also recruited at The First Hospital of Jilin University. Each individual had undergone a medical examination during the period 2010-2011, and all were free from any autoimmune diseases or cancer. All participants in this study provided a written informed consent.

Tissues and blood treatment. Forty-four liver tissue samples from the corresponding cancer patients were resected from the center of the tumor block (20-30 mg in weight and 3-8 $\mathrm{mm}$ in size). Following ice-cold PBS washes, the tumor tissues were split into small pieces for RNA isolation and protein extraction for further immunoblot analyses. All RNA and protein samples were kept in a refrigerator at $-80^{\circ} \mathrm{C}$.

Five $\mathrm{ml}$ of venous blood from each patient was collected and used to extract peripheral blood mononuclear cells (PBMCs). The same procedure was applied to the $10 \mathrm{HC}$.

Cell cultures and transfections. The human liver carcinoma cell line HepG2, obtained from the American Type Culture Collection (ATCC), was cultured in RPMI-1640 containing $10 \% \mathrm{v} / \mathrm{v}$ fetal bovine serum (FBS), and cultivated at $37^{\circ} \mathrm{C}$ in a $5 \% \mathrm{CO}_{2}$ incubator. One day before plasmid transfections, cells $\left(3 \times 10^{6}\right)$ were seeded onto 6-well plates (Iwaki Cell Biology, Tokyo, Japan) at $80 \%$ confluency. Following incubation for $24 \mathrm{~h}$, cells were prepared for transient transfection with $2 \mu \mathrm{g}$ of plasmid DNA/plate, using Lipofectamine ${ }^{\circledR} 2000$ (Invitrogen Life Technologies, Carlsbad, CA, USA), according to the manufacturer's instructions. After this step, cells were incubated in serum- and antibiotic-free culture medium for $6 \mathrm{~h}$, and then cultured with complete medium. Cells were collected at $48 \mathrm{~h}$ following transfection.

Plasmid construction. Human SAMHD1 cDNA (Genbank: NM_015474) was amplified from tissues of the HCC patients by PCR using the WT primer, as shown in Table I. PCR products were then purified and cloned into a pGEM-T vector (Promega Corporation, Madison, WI, USA), and the target monoclones were selected through blue-white colony screenings. The following splice variants of SAMHD1 were identified with sequencing: wild-type (WT), exon4 insertion (ins4), exon8-9 deletion (de18-9), exon13 deletion (del13) and exon14 deletion (del14). The sequence of each variant was excised from the pGEM-T vector by ApaI and NotI (Takara Bio Inc., Shiga, Japan), and inserted into the plasmid pcDNA3.1(-)/Myc-His version A (Invitrogen Life Technologies) to construct pcDNA-His-SAMHD1 WT, ins4, del8-9, del13 and del14, respectively. Stop codons were excluded from the reverse primer of WT SAMHD1 to allow using the His-tag of pcDNA. To exclude the influence of endogenous SAMHD1 on the results, the Vpx gene was amplified from SIVmac239 (using primers listed in Table I) to induce intracellular SAMHD1 degradation. The Vpx start/stop codons were included in the primers, and $X b a \mathrm{I}$ and $A p a \mathrm{I}$ restriction sites were also created for subcloning into pCMV-N-His (Beyotime Institute of Biotechnology Co., Ltd., Shanghai, China). The resulting vector is referred to as pCMV-His-Vpx.

RNA/DNA extractions and RT-PCR. The HepG2 cells were collected after transfection, and total RNA was isolated using TRIzol (Invitrogen Life Technologies) according to the manufacturer's instructions. RNA pellets were ethanol-precipitated, washed, and resuspended in sterile ribonuclease-free water. Reverse transcription was carried out using the Superscript II ${ }^{\circledR}$ enzyme (Gibco-BRL, Carlsbad, CA, USA). The presence of SAMHD1 mutants was checked using the primers shown in Table I.

In order to verify whether the mutations occurred at the level of the genome, we prepared DNA samples using a Universal Genomic DNA Extraction kit (Takara Bio Inc.) and designed primers (Table I) based on the SAMHDI genomic sequence (Genbank: NG_017059). PrimeSTAR HS DNA Polymerase (Takara Bio Inc.) was used in the subsequent PCR reactions, performed following the manufacturer's instructions.

Western blot analysis. Cells were harvested $48 \mathrm{~h}$ after transfection, and lysed in RIPA buffer with protease inhibitors (Roche Diagnostics KK, Basel, Switzerland, USA). Following incubation for $15 \mathrm{~min}$ on ice, cells were centrifuged for $10 \mathrm{~min}$ at $12,000 \mathrm{x} \mathrm{g}$ at $4^{\circ} \mathrm{C}$. The proteins were incubated for 3 min at $100^{\circ} \mathrm{C}$, and then separated on a $10 \%$ SDS-PAGE gel before transferring onto a polyvinylidene difluoride (PVDF) membrane (GE Healthcare). Anti-His monoclonal antibody (BioVision Inc., Mountain View, CA, USA) and anti-GAPDH monoclonal antibody (LifeTein LLC, South Plainfield, NJ, USA) were used for western blot analysis. The secondary antibody used was horseradish peroxidase (Santa Cruz Biotechnology Inc., Santa Cruz, CA, USA) and it was visualized using enhanced chemiluminescence (ECL) (Thermo Fisher Scientific, Jan Jose, CA, USA).

Cell cycle analysis. Following transfections, cells were collected by trypsinization, washed twice with PBS before fixation in $75 \%$ pre-cooled ethanol, and kept at $4^{\circ} \mathrm{C}$ overnight. Following RNaseA $(1 \mu \mathrm{g} / \mathrm{ml})$ treatment for $30 \mathrm{~min}$ at $37^{\circ} \mathrm{C}$, cells were re-suspended with propidium iodide $(50 \mu \mathrm{g} / \mathrm{ml})$, and incubated on ice in the dark for $30 \mathrm{~min}$. The cell cycle distribution was determined by FACSCalibur flow cytometry (BD Biosciences, San Jose, CA, USA) and analyzed using Multicycle software.

Statistical analyses. All the experiments in this study were independently repeated for at least three times. Statistical significance of comparisons between treatments was evaluated using a one-way ANOVA followed by a Tukey's test. A p-value (P) $<0.05$ was considered to indicate a statistically significant difference. 
Table I. Primers designed for vector construction and validation of mutations.

\begin{tabular}{|c|c|c|}
\hline Amplicon & Primer sequence & Length (bp) \\
\hline WT & $\begin{array}{l}\text { F: 5'-ATGCAGCGAGCCGAT-3' } \\
\text { R: 5'-CATTGGGTCATCTTTAA-3' }\end{array}$ & 1,869 \\
\hline Vpx & $\begin{array}{l}\text { F: 5'-TCTAGAGCATGTCAGATCCCAGGGAGAGAAT-3' } \\
\text { R: 5'-GGGCCCGCTTATGCTAGTCCTGGAGGGGG-3' }\end{array}$ & 339 \\
\hline ins 4 & $\begin{array}{l}\text { F: 5'-GATACAATGAAGGAAGAAGTAAAAT-3' } \\
\text { R: 5'-CGGGCGAGCAAGTGG-3' }\end{array}$ & 408 \\
\hline del8-9 & $\begin{array}{l}\text { F: 5'-ACCACTTGAATCACCTGTCG-3' } \\
\text { R: 5'-TAACCTGCGGCTTGGTG-3' }\end{array}$ & 719 \\
\hline del13 & $\begin{array}{l}\text { F: 5'-GGGTTATCAACATGGATTATGG-3' } \\
\text { R: 5'-GAGTTGGATTTTGGACTGAAG-3' }\end{array}$ & 315 \\
\hline del14 & $\begin{array}{l}\text { F: 5'-CCAGGGACTGCCATCATC-3' } \\
\text { R: 5'-GCAGAAGTTGTGAAACATCCA-3' }\end{array}$ & 570 \\
\hline ins4 genomic & $\begin{array}{l}\text { F: 5'-CACTTGAGGTCAGGAGTTCGC-3' } \\
\text { R: 5'-CGATTGTGTGAAGCTCCTGGA-3' }\end{array}$ & 763 \\
\hline del8-9 genomic & $\begin{array}{l}\text { F: 5'-TTGGTGCCTATCCTAAAACTTCC-3' } \\
\text { R: 5'-AAACCCCAAAACCAGTGTCTAAC-3' }\end{array}$ & 598 \\
\hline del13 genomic & $\begin{array}{l}\text { F: 5'-TCAGGCTGGTCTCGAACTCC-3' } \\
\text { R 5'-ACTTTTTCCTCTGTGCTTGTATGC-3' }\end{array}$ & 672 \\
\hline del14 genomic & $\begin{array}{l}\text { F: 5'-GTTCCATTTCTTGTTATGCTCCTAC-3' } \\
\text { R: 5'-GCTGATCTTAAGCTCTTCGTCTC-3' }\end{array}$ & 390 \\
\hline
\end{tabular}

WT, wild-type; F, forward; R, reverse; ins4, exon4 insertion; del8-9, exon8-9 deletion; del13, exon13 deletion; del14, exon14 deletion.

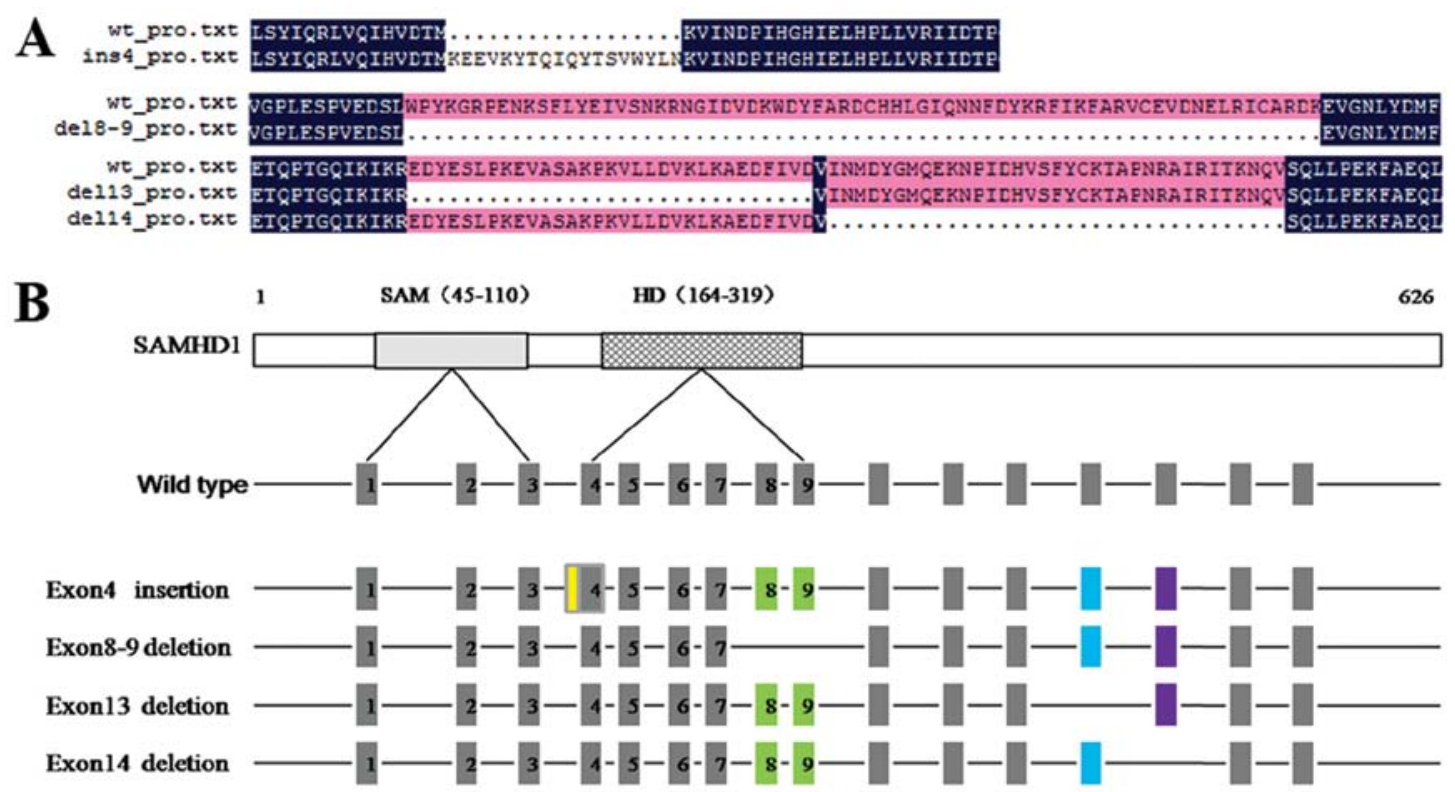

Figure 1. Types of SAMHD1 mutations occurring in liver cancer patients. (A) After PCR products of SAMHD1 were cloned into the pGEM-T vector, and target monoclones were selected by blue-white screenings and sequencing, an insertion in exon4 and deletions of exons8-9, 13 and 14 were identified in liver cancer patients. (B) The genomic location of SAMHD1 mutations, displayed in a bar chart.

\section{Results}

Natural SAMHD1 splice variants in HCC. Deletions of exon8-9 and 14 have been identified in a variety of cell types, including 293T, THP-1 and primary human CD4 ${ }^{+} \mathrm{T}$ cells. It is very likely that the deleted variants are naturally expressed along with the full-length SAMHD1 (13). In order to determine whether these variants also occurr in liver cancer, 44 tissues from patients with HCC were tested. We identified, by sequencing, a natural insertion in exon4 and deletions in exons8-9, 13 and 14 (Fig. 1). 
Table II. Clinical data of liver cancer patients and healthy controls.

\begin{tabular}{lcccc}
\hline Parameters & $\begin{array}{c}\text { HBV-infected } \\
\text { patients }(\mathrm{n}=22)\end{array}$ & $\begin{array}{c}\text { HCV-infected } \\
\text { patients }(\mathrm{n}=10)\end{array}$ & $\begin{array}{c}\text { Non-virus-infected } \\
\text { patients }(\mathrm{n}=12)\end{array}$ & HC $(\mathrm{n}=10)$ \\
\hline Mean age (range), years & $54(37-68)$ & $60(55-66)$ & $62(44-66)$ & $35(28-42)$ \\
Gender (male/female) & $16 / 6$ & $7 / 3$ & $7 / 3$ & $8.29(1.74-121.3)$ \\
Mean AFP (range), ng/ml & $70.64(1.3-3,430)$ & $21.57(11.14-325.4)$ & $32(15-38)$ & $18(15-23)$ \\
Mean AST (range), U/1 & $40(13-75)$ & $53.8(21-68)$ & $21(9-50)$ & $20(14-28)$ \\
Mean ALT (range), U/1 & $39(12-65)$ & $41(24-94)$ & $25 \%(3 / 12)^{\mathrm{a}}$ & $10 \%(1 / 10)$ \\
ins4 frequency & $36.4 \%(8 / 22)^{\mathrm{a}, \mathrm{b}}$ & $30 \%(3 / 10)^{\mathrm{a}, \mathrm{b}}$ & & $10.21)$ \\
\hline
\end{tabular}

${ }^{a}$ Statistically significant difference compared to the healthy controls $(\mathrm{P}<0.01)$; ${ }^{\mathrm{b}}$ statistically significant difference compared to the non-virus-infected group $(\mathrm{P}<0.01)$. HBV/HCV, hepatitis $\mathrm{B} / \mathrm{C}$ virus; $\mathrm{HC}$, healthy controls; ins4, exon4 insertion.

A

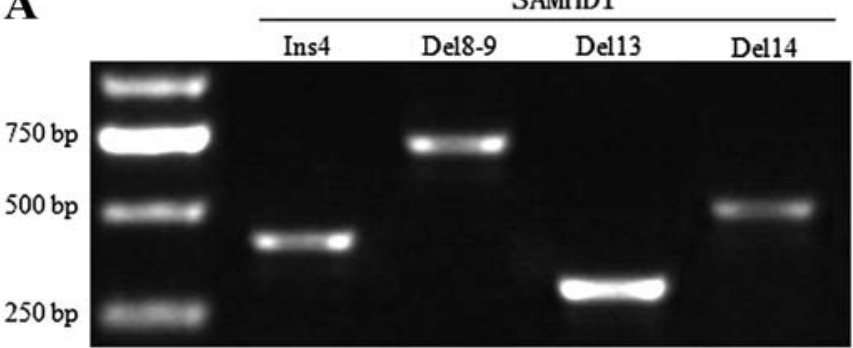

B

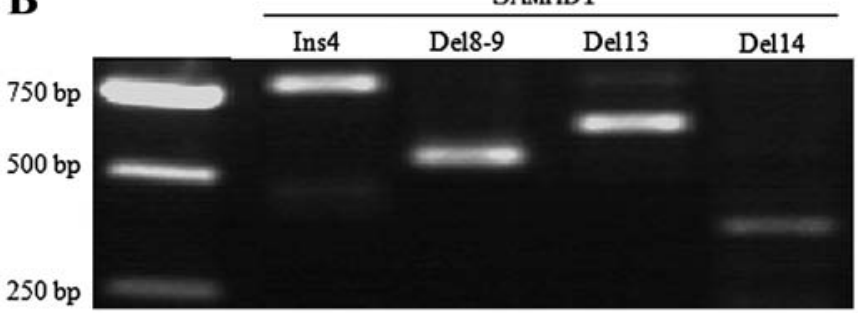

Figure 2. Expression level of SAMHD1 splice variants. (A) SAMHD1 splice variants were amplified on RNAs from positive samples by PCR (ins4, 408 bp; del8-9, 719 bp; del13, 315 bp; del14, 570 bp). Primers were designed based on the principle of amplifying inserted sequences or sequences spanning the junction of deletions, so as to increase specificity. (B) PCR products of genomic DNA purified from positive patients identified as possessing the mutation at the transcriptional level (ins 4 genomic, $763 \mathrm{bp}$; del8-9 genomic, 598 bp; del13 genomic, 672 bp; del14 genomic, 390 bp). Primers amplified sequences adjacent to the mutations.

Frequency of splice variants in patients and controls. Among the identified splice variants, deletions of exon8-9, 13 and 14 existed naturally in both the HCC and the controls, but the insertion of exon4, which contained $62 \mathrm{bp}$, occurred more frequently in the HCC and was seldom found in the HC. As shown in Table II, the frequency of exon4 insertion was $36.4 \%$ and $30 \%$ in the $\mathrm{HBV} / \mathrm{HCV}$-infected groups respectively, while it was $25 \%$ in the non-virus-infected group and only $10 \%$ in the $\mathrm{HC}$. The high frequency of the insertion in the virus-infected groups compared to the non-virus-infected and HC groups was statistically significant $(\mathrm{P}<0.01)$, while the frequencies of the deletions showed no difference in these groups (data not shown). These results suggest that the exon4 insertion might correlate with the occurrence of virus-infected HCC.
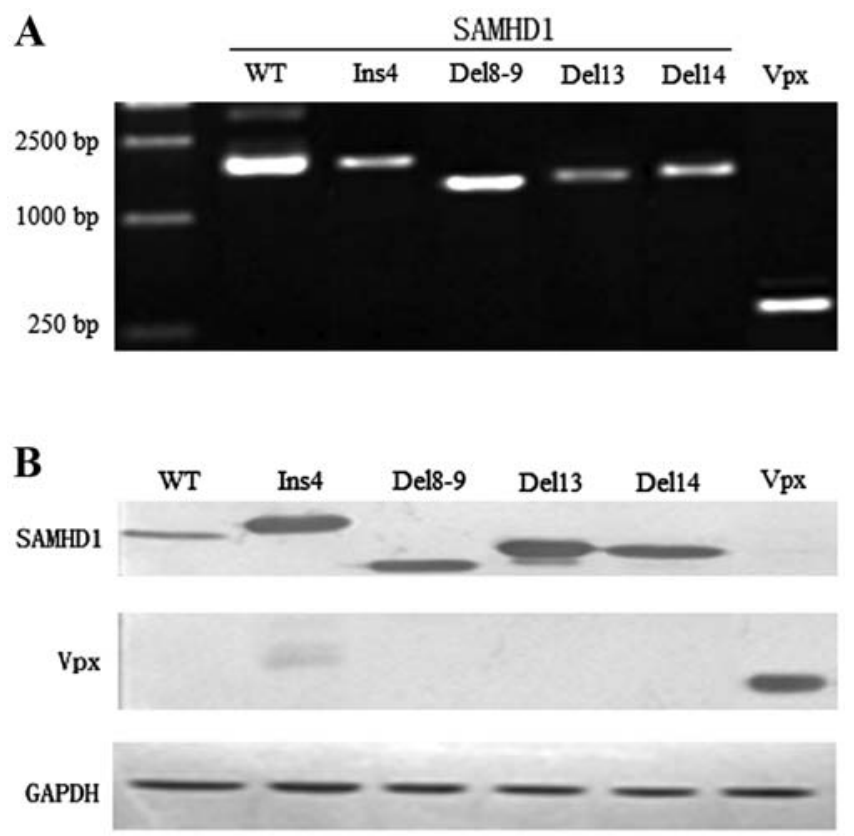

Figure 3. Construction of vectors bearing the mutations. (A) PCR products of HepG2 cells that were co-transfected with pcDNA-SAMHD1 WT, ins4, del8-9, del13, del14 and Vpx respectively (WT, 1,869 bp; ins4, 1,932 bp; del8-9, 1,659 bp; del13, 1,776 bp; del14, 1,764 bp; Vpx: 339 bp). (B) Whole-cell extracts were prepared at $48 \mathrm{~h}$ following transfection and separated on $10 \%$ SDS-PAGE. Immunoblot analysis was performed with Anti-His monoclonal antibodies specific to SAMHD1, Vpx and GAPDH (WT, $75 \mathrm{kDa}$; ins4, $78 \mathrm{kDa}$; del8-9, $68 \mathrm{kDa}$; del13, $72 \mathrm{kDa}$; del14, $71 \mathrm{kDa}$; Vpx, $16 \mathrm{kDa}$; GAPDH, $37 \mathrm{kDa})$.

Confirmation of the expression profile of SAMHD1 splice variants. In order to investigate whether the natural variants also occur at the genomic level, we used the genomic DNAs extracted from the positive samples to test the expression of the corresponding mutations. As shown in Fig. 2, the above mutations occurred at the transcriptional level, but not at the genomic level.

Investigation of the different roles of SAMHD1 splice variants in regulating cell sensitivity to cisplatin. To investigate the effect of splice variants in HCC, we selected pcDNA3.1(-)/Myc-His to construct plasmids of the different SAMHD1 mutants (pcDNA-His-SAMHD1 WT, ins4, 


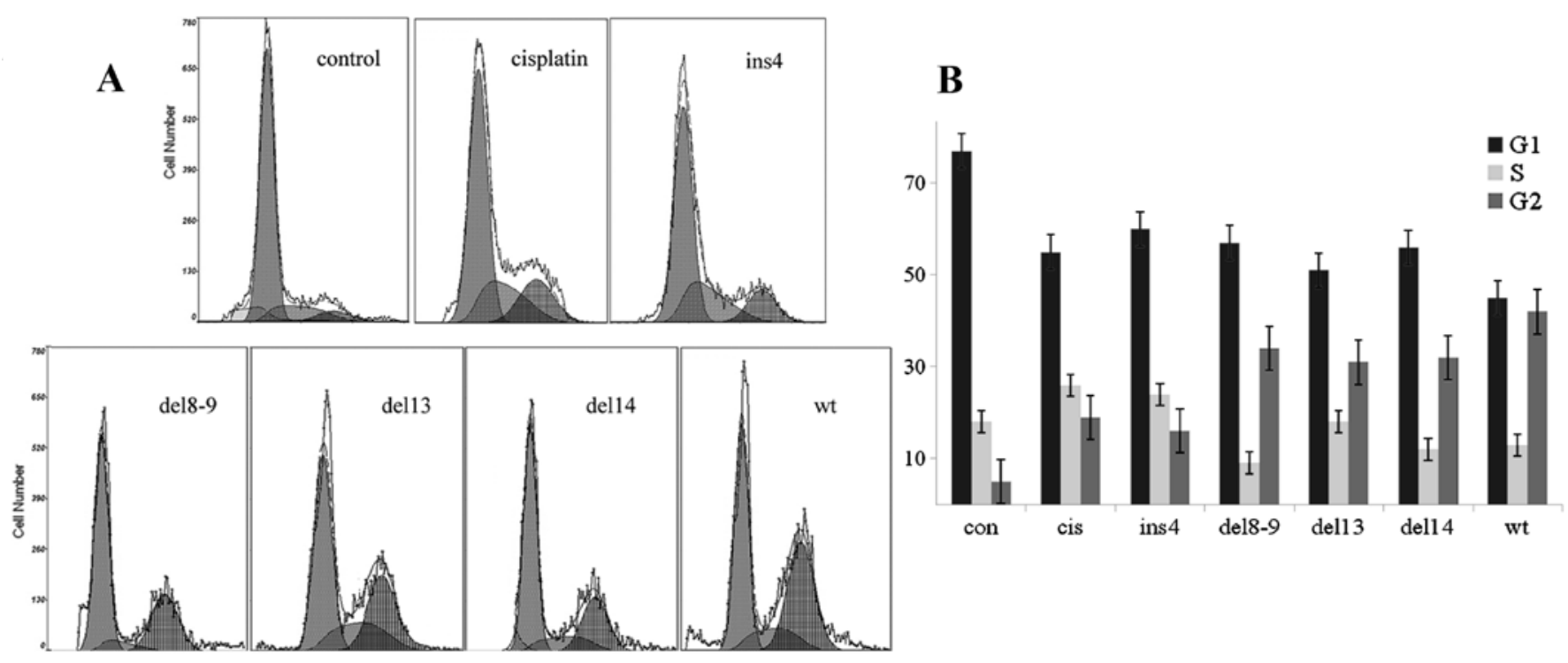

Figure 4. Variation in cell cycle distribution following cisplatin treatment. Each variant was co-transfected with pCMV-His-Vpx and pcDNA-His-SAMHD1 WT, ins4, del8-9, del13 and del14 respectively, while the control and the cisplatin groups were co-transfected with pCMV-His-Vpx and pcDNA3.1(-)/Myc-His. Following co-transfection, cells were treated with $1 \mu \mathrm{M}$ cisplatin except for the control group, and analyzed by FACS $18 \mathrm{~h}$ later. Results are presented as percent distribution of cells in different phases of the cell cycle.

del8-9, del13 and del14) and used pCMV-N-His to construct a pCMV-His-Vpx vector. Next, we co-transfected HepG2 with pCMV-His-Vpx and vectors of the SAMHD1 mutants, respectively, as shown in Fig. 3. Following an 18-h transfection with $1 \mu \mathrm{M}$ cisplatin (15), we examined the effects of SAMHD1 splice variants in regulating cell sensitivity to cisplatin. The results indicated that the WT SAMHD1 had the strongest ability to increase cell sensitivity to the drug, while the ability of del8-9, del13 and del14 was relatively weaker. As shown in Fig. 4, there was no obvious difference between the ins 4 and the single-cisplatin-treated group.

\section{Discussion}

Previous studies have shown that SAMHD1, along with other members of the same family such as TREX1 and RNase H2, which are characterized by a 3'-5' exonuclease activity, have effects on autoimmune diseases, auch as AGS and SLE. This is related to mutations in these proteins that lead to nucleic acid accumulation and genomic instability $(3,15)$. In the present study, we identified several natural slice variants of SAMHD1 from 44 patients with $\mathrm{HCC}$ and 10 healthy controls, including variants with an insertion in exon 4 and deletions of exon8-9, 13 and 14. It was further confirmed that these mutations occurred at the transcriptional level and not at the genomic level.

The deletion of exon 13 has been shown to cause cerebral vasculopathy and early onset of cerebrovascular accidents (13). The exon8-9 and 14 deletion variants have been reported to be expressed along with the full-length SAMHD1 in a variety of cell types (14). In the present study, we found that del8-9, del13 and del14 were generally present in both patients and $\mathrm{HC}$, whereas the exon 4 insertion showed a high prevalence in virus-infected HCC. A total of 14 samples out of 44 HCC patients had the exon4 insertion, resulting in a frequency of $36.4 \%$ in the HBV-infected group, 30\% in the HCV-infected group, $25 \%$ in the non-virus-infected group and $10 \%$ in $\mathrm{HC}$, respectively. There was a statistically significant difference between the virus-infected groups compared to the non-virus-infected group and the HC $(\mathrm{P}<0.01)$. These results indicate a potential relationship between the SAMHD1 exon4 insertion and the occurrence of virus-induced HCC.

Differences in activities among SAMHD1 splice variants were also investigated, so as to understand their roles in cancer. In addition to the complete SAMHD1 gene composed of 16 exons, we constructed eukaryotic expression vectors by conjugating pcDNA3.1(-)/Myc-His with ins4, del8-9, del13 and del14 respectively. To exclude the influence of endogenous SAMHD1 on the results, pCMV-His-Vpx was used to degrade the intracellular SAMHD1. After co-transfecting HepG2 with the above expression vectors, the typical antineoplastic drug cisplatin was added into each pore to inspect the different effects of SAMHD1 slice variants. The result showed that the WT SAMHD1 vector induced G2 arrest (Fig. 4B) and had the strongest ability to increase the cell sensitivity to cisplatin, while del8-9, 13 and 14 were relatively weaker. Ins4 and the single-cisplatin-treated group presented the weakest activity, and showed no obvious differences between them. By sequence alignments, a stop codon (TAG) was identified in ins4, leading to a translation-terminating mutation. This might explain the similarity between the ins 4 and the single-cisplatin-treated group with regards to $\mathrm{G} 2$ arrest.

The different activities of SAMHD1 slice variants in regulating cell sensitivity to medicinal treatment suggest that SAMHD1 is not only an effector in the innate immune response, but might also be a negative regulator during the occurrence of HCC. We presumed that a full-length SAMHD1 might act as an antitumor factor by increasing the cell sensitivity to chemotherapy drugs. However in the presence of other splice variants, the antitumor activity of SAMHD1 might be gradually weakened, leading to a high risk of liver 
cancer. Although the splice variants of the gene SAMHDI are naturally present in vivo, they displayed different abilities to increase cell susceptibility to cisplatin. Because of its high frequency in the virus-infected groups, the exon4 insertion, which led to an abnormal SAMHD1 translation termination, appears to correlate with the risk of liver cancer. Although the results indicated that this insertion might be an indicator of hepatocarcinogenesis, the precise mechanism behind the occurrence of this insertion still needs to be studied in detail.

\section{Acknowledgements}

We are very grateful to Dr Ding, Dr Zhang and Dr Yu for the sample collection and the helpful clinical information support. This study was supported by grants from the Special Funded Project of Jilin Provincial Finance Department (no. 2012Z001).

\section{References}

1. Li N, Weiping $\mathrm{Z}$ and Cao X: Identification of human homologue of mouse IFN- $\gamma$ induced protein from human dendritic cells. Immunol Lett 74: 221-224, 2000.

2. Crow MK, Kirou KA and Wohlgemuth J: Microarray analysis of interferon-regulated genes in SLE. Autoimmunity 36: 481-490, 2003.

3. Rice GI, Bond J, Asipu A, Brunette RL, Manfield IW, Carr IM, Fuller JC, et al: Mutations involved in Aicardi-Goutières syndrome implicate SAMHD1 as regulator of the innate immune response. Nat Genet 41: 829-832, 2009.

4. Ramantani G, Kohlhase J, Hertzberg C, Innes AM, Engel K, Hunger S, Borozdin W, et al: Expanding the phenotypic spectrum of lupus erythematosus in Aicardi-Goutières syndrome. Arthritis Rheum 62: 1469-1477, 2010.

5. Powell RD, Holland PJ, Hollis $\mathrm{T}$ and Perrino FW: Aicardi-Goutières syndrome gene and HIV-1 restriction factor SAMHD1 is a dGTP-regulated deoxynucleotide triphosphohydrolase. J Biol Chem 286: 43596-43600, 2011.
6. Goldstone DC, Ennis-Adeniran V, Hedden JJ, Groom HC, Rice GI, Christodoulou E, Walker PA, et al: HIV-1 restriction factor SAMHD1 is a deoxynucleoside triphosphate triphosphohydrolase. Nature 480: 379-382, 2011.

7. Lahouassa H, Daddacha W, Hofmann H, Ayinde D, Logue EC, Dragin L, Bloch N, et al: SAMHD1 restricts the replication of human immunodeficiency virus type 1 by depleting the intracellular pool of deoxynucleoside triphosphates. Nat Immunol 13: 223-228, 2012

8. Kao JH and Chen DS: Global control of hepatitis B virus infection. Lancet Infect Dis 2: 395-403, 2002.

9. Daga PR, Duan J and Doerksen RJ: Computational model of hepatitis B virus DNA polymerase: Molecular dynamics and docking to understand resistant mutations. Protein Sci 19: 796-807, 2010.

10. Lindenbach BD and Rice CM: Unravelling hepatitis $C$ virus replication from genome to function. Nature 436: 933-938, 2005.

11. Alter MJ, Kruszon-Moran D, Nainan OV, McQuillan GM, Gao F, Moyer LA, Kaslow RA, et al: The prevalence of hepatitis C virus infection in the United States, 1988 through 1994. N Engl J Med 341: 556-562, 1999.

12. Murayama A, Weng L, Date T, Akazawa D, Tian X, Suzuki T, Kato $\mathrm{T}$, et al: RNA polymerase activity and specific RNA structure are required for efficient $\mathrm{HCV}$ replication in cultured cells. PLoS Pathog 6: e1000885, 2010.

13. Ramesh V, Bernardi B, Stafa A, Garone C, Franzoni E, Abinun M, Mitchell $\mathrm{P}$, et al: Intracerebral large artery disease in Aicardi-Goutières syndrome implicates SAMHD1 in vascular homeostasis. Dev Med Child Neurol 52: 725-732, 2010.

14. Welbourn S, Miyagi E, White TE, Diaz-Griffero F and Strebel K: Identification and characterization of naturally occurring splice variants of SAMHD1. Retrovirology 9: 86, 2012.

15. He G, Kuang J, Khokhar AR and Siddik ZH: The impact of S- and G2-checkpoint response on the fidelity of G1-arrest by cisplatin and its comparison to a non-cross-resistant platinum (IV) analog. Gynecol Oncol 122: 402-409, 2011. 